\title{
Experimental station for multiscale surface structural analyses of soft-material films at SPring-8 via a GISWAX/GIXD/XR-integrated system
}

\author{
Hiroki Ogawa ${ }^{1}$, Hiroyasu Masunaga ${ }^{1}$, Sono Sasaki ${ }^{1}$, Shunji Goto ${ }^{1}$, Takashi Tanaka ${ }^{2}$, Takamitsu Seike ${ }^{1}$, \\ Sunao Takahashi ${ }^{1}$, Kunikazu Takeshita ${ }^{1}$, Nobuteru Nariyama ${ }^{1}$, Haruhiko Ohashi ${ }^{1}$, Toru Ohata ${ }^{1}$, \\ Yukito Furukawa $^{1}$, Tomohiro Matsushita ${ }^{1}$, Yasuhide Ishizawa ${ }^{1}$, Naoto Yagi $^{1}$, Masaki Takata ${ }^{1,2,3}$, \\ Hideo Kitamura ${ }^{2}$, Atsushi Takahara ${ }^{4}$, Kazuo Sakurai ${ }^{4}$, Kohji Tashiro ${ }^{4}$, Toshiji Kanaya ${ }^{4}$, Yoshiyuki Amemiya ${ }^{4}$, \\ Kazuyuki Horie ${ }^{4}$, Mikihito Takenaka ${ }^{4}$, Hiroshi Jinnai ${ }^{4}$, Hiroshi Okuda ${ }^{4}$, Isamu Akiba ${ }^{4}$, Isao Takahashi ${ }^{4}$, \\ Katsuhiro Yamamoto $^{4}$, Masamichi Hikosaka ${ }^{4}$, Shinichi Sakurai ${ }^{4}$, Yuya Shinohara ${ }^{4}$, Yasunori Sugihara ${ }^{5}$ \\ and Akihiko Okada ${ }^{5}$
}

To gain a better understanding of the function of soft-material thin films, one should investigate the multiscale structural information from the surface roughness down to the atomically truncated structures at microarea. To achieve such an integrated investigation, a new experimental system has been launched by coupling with the measurement techniques, which include grazing incidence small/wide-angle X-ray scattering (GISWAXS) and grazing incidence X-ray diffraction (GIXD), as well as X-ray reflectivity (XR), at the BL03XU beamline of SPring-8. The high brilliance and low divergence beam allows the surface and interface structure measurements from the angstrom to the micrometer scale using a soller-slit system for proper optimization of measurement precision. A typical structural analysis of the soft-material film was performed to evaluate the practical performance and specifications of the experimental system.

Polymer Journal (2013) 45, 109-116; doi:10.1038/pj.2012.194; published online 14 November 2012

Keywords: FSBL; grazing incidence small/wide-angle X-ray scattering; grazing incidence X-ray diffraction; polymer thin films; SPring-8; X-ray reflectivity

\section{INTRODUCTION}

Soft-material thin films have attracted much attention because of their wide variety of applications, such as in coatings, adhesives, surface friction, lubricants and dielectric layers. Many studies on polymer thin films have been performed to reveal their properties. ${ }^{1-4}$ It is now considered that the physical properties of polymer thin films (for example, phase separation or glass transition temperature) are strongly influenced by the surface and interface. ${ }^{5-7}$ Clarification of the relationship between the morphological characteristics, including hierarchy and physical properties, is, therefore, indispensable in developing new functions for soft-material thin films. The investigations of the morphological characteristics have hitherto been performed using transmission electron microscopy, atomic force microscopy and optical microscopy, giving access to scales ranging from nanometers to micrometers. These analytical tools provide an accurate but local view of the surface morphology. Transmission small-angle X-ray scattering (SAXS) is a very powerful tool as a nondestructive technique to obtain the structural information from a wide variety of samples. Owing to the high photon flux, time-resolved or in situ experiments during a formation process can be conducted at third-generation synchrotron radiation sources using the insertion devices provided.

Grazing incidence small/wide-angle X-ray scattering (GISWAXS) is the combination of a grazing incidence (GI) reflection setup and SAXS for characterizing the morphological characteristics of soft materials in thin films. X-ray reflectivity (XR) measurement provides invaluable information about the films, for example, thickness. Grazing incidence X-ray diffraction (GIXD) measurements offer the opportunity to determine the heterogeneous surface and interface structures in the in-plane direction. ${ }^{8-10}$ GIXD is also a sensitive tool for measuring the crystallization and/or molecular orientation of thin films. Therefore, an integrated investigation with GISWAX, GIXD and XR can be used together to measure the hierarchal soft-material thin films

\footnotetext{
1Japan Synchrotron Radiation Research Institute (JASRI/SPring-8), Sayo, Hyogo, Japan; ${ }^{2}$ RIKEN SPring-8 Center, Sayo, Hyogo, Japan; ${ }^{3} T h e$ University of Tokyo, Tokyo, Japan; ${ }^{4}$ FSBL (Representatives of the academic members), Sayo, Hyogo, Japan and 5FSBL (Representatives of the industrial members), Sayo, Hyogo, Japan

Correspondence: Dr H Ogawa, Research and Utilization Division, Japan Synchrotron Radiation Research Institute (JASRI/SPring-8), 1-1-1 Kouto, Sayo, Hyogo 679-5198, Japan. E-mail: ogawa@spring8.or.jp

Received 3 September 2012; revised 24 September 2012; accepted 25 September 2012; published online 14 November 2012
} 
from the angstrom surface roughness to the micrometer structure. Each measurement of GISWAXS, GIXD and XR also has an important role in clarifying the structural information with hierarchical characteristics.

Recently, a beamline dedicated to the structural analysis of soft materials was built at the BL03XU FSBL (advanced soft-material beamline) of SPring- 8 . The BL03XU beamline has two experimental hutches: the first hutch is $6.0 \mathrm{~m}$ long, $4.0 \mathrm{~m}$ wide and $4.0 \mathrm{~m}$ high, and is designed for GISWAXS, GIXD and XR using the compact multiaxis diffractometer, and the second hutch is $10.0 \mathrm{~m}$ long, $5.0 \mathrm{~m}$ wide and $4.5 \mathrm{~m}$ high, and is designed for transmission SAXS and transmission WAXS measurements. Because of the high photon flux at thirdgeneration synchrotron radiation sources that have an undulator, one of the aims of the first hutch is to clarify the formation process of the hierarchical surface and interface structure in soft-material thin films. The specification of the optics, the second hutch and the FSBL consortium is fully described elsewhere. ${ }^{11}$ Here, we report the characteristics and performance of the new instrument, the 'nanoscopic surface and interface measurement system', in the first hutch.

\section{EXPERIMENTAL PROCEDURES}

\section{Instrument components}

Beamline design. Figure 1 shows the outline of the beamline designed for the GISWAXS/GIXD/XR measurements of the surface and interface structures of soft-material thin films. X-rays from the standard-type SPring- 8 in-vacuum undulator are monochromated by means of a silicon (Si) (1 111$)$ double-crystal monochromator (Figure 1A) with a band pass of $\Delta E / E \sim 10^{-4}$ (available energy: 5-18 keV). A pair of perpendicularly aligned Kirkpartrick-Baez mirrors (Figure $1 \mathrm{~b}$ ), located $53.2 \mathrm{~m}$ from the light source, are used to focus the X-ray beam. The intensity of the X-ray beam is measured by an ionization chamber current (Ohyo Koken Kogyo, Saitama, Japan; S-1329A), and the X-ray beam is attenuated to a suitable intensity for the measurements by the absorbers located at point C in Figure 1. An X-ray shutter (Vincent Associates, Rochester, NY, USA; UNIBLITZ XRS6) and a quadrant XY slit (Kohzu Precision, Kanagawa, Japan; GHM-13B), which are used for reducing parasitic scattering from the mirrors and for optimizing the beam size, are also installed at this point. The guard pinhole for reducing parasitic scattering generated by the quadrant XY slit and the ionization chamber for measuring the effective intensity of the incident X-ray beam are placed at point D in Figure 1. The diffractometer is set so that the sample position is $3.5 \mathrm{~m}$ from the KirkpartrickBaez mirrors; the focusing ratio is expected to be $1 / 15$, taking into account the distance between the light source and the Kirkpartrick-Baez mirrors $(53.2 \mathrm{~m})$. The minimum size of the X-ray beam at the sample position is nearly $10 \times 10 \mu \mathrm{m}^{2}$ (horizontal $\times$ vertical) full-width at half-maximum with a photon flux of $10^{12}$ photons per $s$.

System overview. The 'nanoscopic surface and interface measurement system' in the first hutch that consists of two main components: the diffractometer (Figure 1E) and the detecting system include a vacuum path and detectors on an optical bench. Figure 2 shows a typical setup for the GISAXS measurements. The setup is systematically designed so that all the methods of the GISWAXS, GIXD and XR experiments are compatible. This is an advantage when compared with setups dedicated using transmission SAXS. In addition to the GISWAXS/GIXD/XR-compatible system, a linkage arrangement of the vacuum path in the first hutch and the second hutch achieves an extremely long camera length, approximately $11.7 \mathrm{~m}$, and a smart grazing incidence ultra-small-angle X-ray scattering measurement system uses the detecting system installed in the second hutch. Details of the grazing incidence ultrasmall-angle X-ray scattering system and outcomes will be described elsewhere.

Furthermore, the first hutch is also equipped with an exhaust system with a large capacity of approximately $1 \mathrm{~m}^{3} \mathrm{~s}^{-1}$ to allow the use of organic solvents inside the hutch, for example, for in situ experiments on the solution-cast process or on the interface between liquid and solid states.

Multiaxis diffractometer system. A compact multiaxis diffractometer $\left(1.4 \times 1.6 \times 1.9 \mathrm{~m}^{3}\right)$ is composed of two sample rotation axes, $\theta_{\mathrm{H}}$ (around the horizontal axis) and $\theta_{\mathrm{V}}$ (around the vertical axis), and two detector arm axes, $\theta_{\mathrm{H}}^{\prime}$ (around the horizontal axis) and $\theta_{\mathrm{V}}^{\prime}$ (around the horizontal axis), as shown in Figure 3 . The angle $\theta_{\mathrm{H}}$ can rotate from $0^{\circ}$ to $30^{\circ}$, with an angular resolution of $0.05 \mathrm{arcsec}$ per pulse, and the angle $\theta_{\mathrm{V}}$ rotates from $-180^{\circ}$ to $180^{\circ}$, with an angular resolution of $0.8 \mathrm{arcsec}$ per pulse. Both the angles $\theta_{\mathrm{H}}^{\prime}$ and $\theta^{\prime}{ }_{\mathrm{V}}$ can cover the angular range from $0^{\circ}$ to $70^{\circ}$, with an angular resolution of 0.09 arcsec per pulse. Consequently, diffraction from the in-plane and outof-plane directions can be measured without changing the sample setting using the multiaxis diffractometer.

The position and the tilt angle of the sample can be precisely controlled with two translation axes $\left(X_{\mathrm{S}}, Y_{\mathrm{S}}\right)$ and two swivel angles $\left(R_{\mathrm{X}}, R_{\mathrm{Y}}\right)$, respectively. The $X_{\mathrm{S}}$ and $Y_{\mathrm{S}}$ translation axes across the direct beam cover from -12.5 to $12.5 \mathrm{~mm}$, with a resolution of $0.1 \mu \mathrm{m}$ per pulse. The height $\left(Z_{\mathrm{S}}\right)$ axis covers from -2.5 to $2.5 \mathrm{~mm}$, with a resolution of $0.025 \mu \mathrm{m}$ per pulse to enable positioning of the sample surface in the direct beam. Both $R_{\mathrm{X}}$ and $R_{\mathrm{Y}}$ scan across the direct beam, providing precise collimation, and rotate from $-5^{\circ}$ to $5^{\circ}$, with an angular resolution of 0.8 arcsec per pulse. In the standard setup, the temperature control system under a vacuum condition of approximately $1.0 \times 10^{1} \mathrm{~Pa}$ is set on the sample stage. The sample temperature is controlled by the heater and the water-cooling chiller from 10 to $200^{\circ} \mathrm{C}$ at the sample surface. For these specific experiments, equipment $500(X) \times 500(Y) \times 70$ $(Z) \mathrm{mm}^{3}$ in size and $4.0 \mathrm{~kg}$ in weight can be mounted directly onto the sample stage.

The in-plane and out-of-plane GIXD measurements are performed with a cerium scintillator (Ohyo Koken Kogyo; KX-101B), which is set on the detector arm. The XR measurements are performed using the scintillator and an Si positive-intrinsic-negative (PIN)-photodiode (Ohyo Koken Kogyo; S-2500) selectively. The PIN-photodiode is needed to protect the scintillator from the intense specularly reflected beam around the critical angle. On the detector arm, therefore, an additional vertical stage is added to allow a rapid change in position between the scintillator and the PIN-photodiode.

The soller slit and two detector slit are used to reduce the parasitic scattering and to improve the angular resolution. A soller slit (HUBER, Bavaria, Germany; 3030.30) installed in front of the detectors determines a divergence angle of $0.099^{\circ}$. The aperture size of the two detector slits is $2.0 \times 0.10 \mathrm{~mm}^{2}$ (horizontal $\times$ vertical), with a divergence angle of $0.01^{\circ}$.

Two-dimensional detecting system. An X-ray image intensifier with a cooled charge-coupled device (CCD) (II + CCD) (Hamamatsu Photonics, Shizuoka, Japan; V7739P + ORCA R2) ${ }^{12}$ and an imaging plate (IP) system (Rigaku, Tokyo, Japan; R-AXIS IV ++ ), as well as a beam monitor (Hamamatsu Photonics; AA40), are used for measuring the GISAXS and grazing incidence wide-angle $\mathrm{x}$-ray scattering (GIWAXS) patterns. Three detectors are set on a

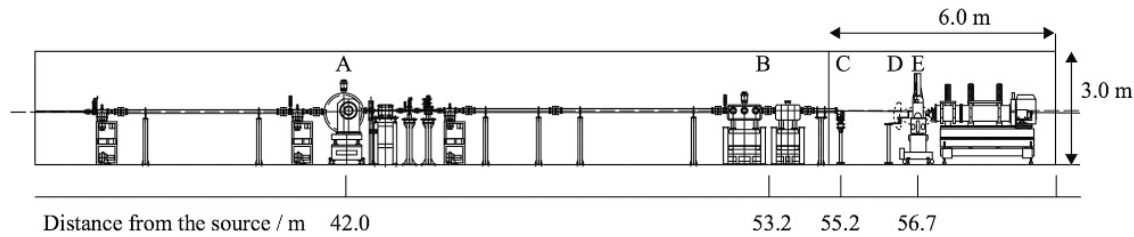

Figure 1 Layout of the optics and the first hutch in the BLO3XU beamline: (A) double-crystal monochromator; (B) Kirkpatrick-Baez mirrors; (C) ionization chamber, absorbers, shutter, slit; (D) guard pinhole; and (E) diffractometer. 


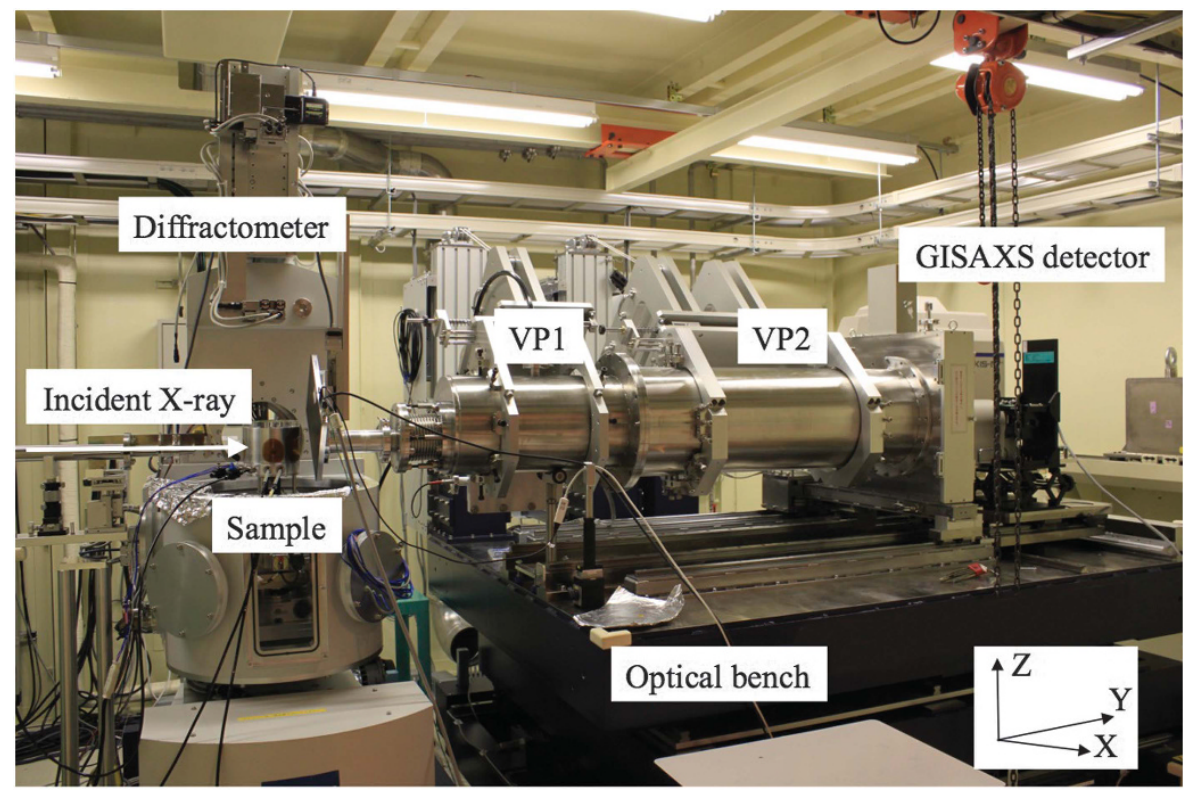

Figure 2 Photograph of the grazing incidence small/wide-angle X-ray scattering (GISAXS) measurement as observed from the side. The camera length is $2018 \mathrm{~mm}$.

a

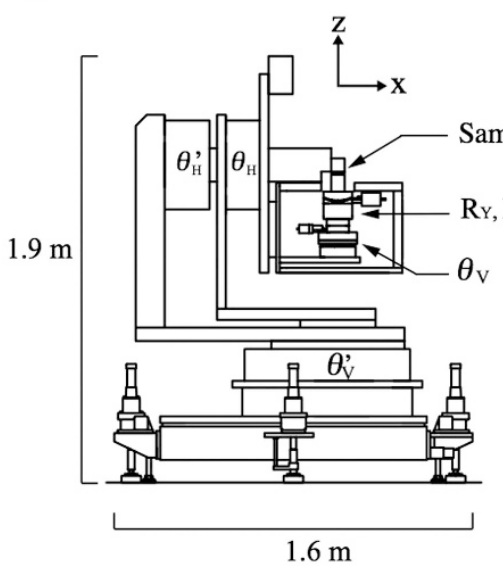

b
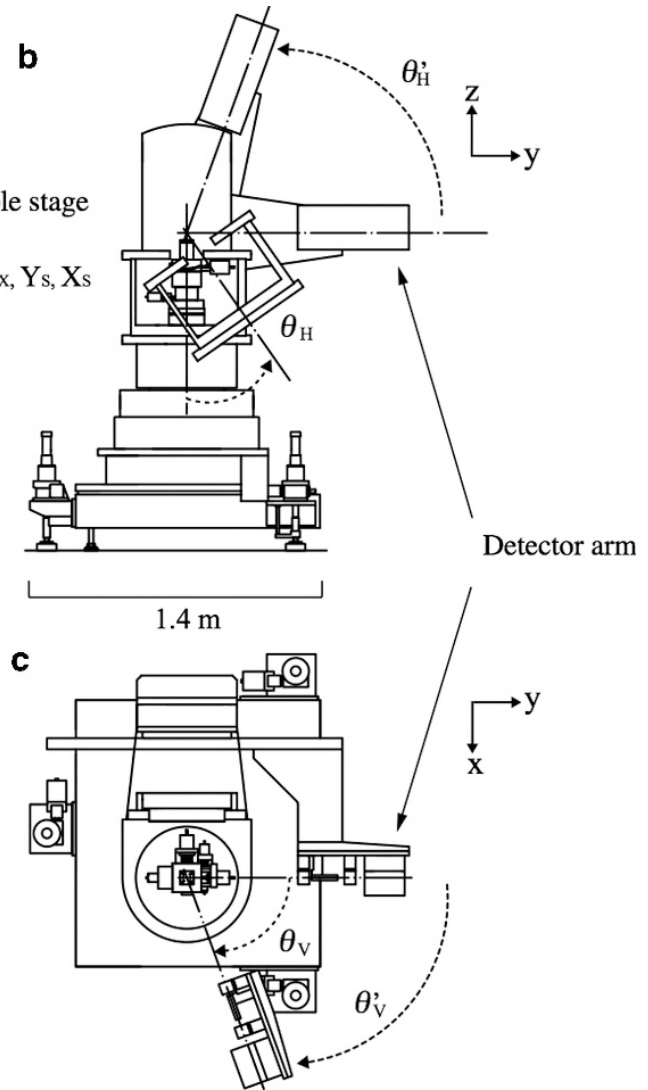

Figure 3 Sketch of the diffractometer from the (a) upstream, (b) side and (c) top view.

stage platform on the optical bench to move them perpendicular (X direction) and parallel (Y direction) to the beam. The movement along the $\mathrm{X}$ direction changes the detectors within several tens of seconds, and the movement along the $\mathrm{Y}$ direction allows measurements with different camera lengths, as described in detail below.
Both the CCD and IP, as well as the beam stop chamber, can be moved along the Yaxis on the optical bench to change the camera length. The rails for the stage platform and the beam stop chamber are parallel to the optical axis (Y direction), with an accuracy $>100 \mu \mathrm{m}$ in the X direction on the optical bench. In the standard setup, three camera lengths of $0.5,1.0$ and $2.0 \mathrm{~m}$ are 
used to cover the $q$ range from 0.05 to $6.0 \mathrm{~nm}^{-1}$ for the CCD detector and from 0.05 to $25.0 \mathrm{~nm}^{-1}$ for the IP detector, with a resolution of $\Delta q / q \approx 10^{-2}$. To reduce the scattering from the air, vacuum paths of $0.5 \mathrm{~m}$ (VP1) and $1.0 \mathrm{~m}$ (VP2) in length can be used for the measurements that use a camera length of 1.0 and $2.0 \mathrm{~m}$, respectively. For the simultaneous measurements of the GIWAXS and the GISAXS, a flat panel detector (Hamamatsu Photonics; C9827DK-10 $)^{13}$ was utilized behind the sample position to cover a quadrant in the $q$ range of $5-30 \mathrm{~nm}^{-1}$ for the GIWAXS measurements.

Table 1 summarizes the specifications of each measurement at the wavelength $\lambda$ of $1.0 \AA$ for typical experiments.

A precise and quick sample alignment with the soller slit. In the GISWAXS and GIXD experiments, the incident beam impinges at a very shallow angle approximately $0.1^{\circ}$ onto the sample surface. Therefore, precise collimation of the incident X-ray beam in the direction parallel to the sample surface is required. For a precise and quick sample setting, a new soller slit and the PINphotodiode system are installed in the VP1 (Figure 4). The homemade soller slit consists of three quartz plates: the upper and lower spaces between the quartz plates are 200 and $40 \mu \mathrm{m}$, with a divergence angle of $0.033^{\circ}$ and $0.164^{\circ}$, respectively (Figure $4 \mathrm{a})$. The relative height $\left(h_{\mathrm{S}}\right)$ and rotation angle $\left(\phi_{\mathrm{S}}\right)$ of the soller slit and the PIN-photodiode to the incident beam are controlled in the range of -50 to $50 \mathrm{~mm}$ and $-10^{\circ}$ to $10^{\circ}$, relatively.

For alignment of the sample surface to the incident beam, this system is set to $h_{\mathrm{S}}=0 \mathrm{~mm}$ and $\phi_{\mathrm{S}}=0^{\circ}$ to detect the direct beam (Figure 4c). In the GISWAXS measurement, this system can avert from the direct beam and the $\mathrm{X}$-ray scattering from the sample as shown in Figure $4 \mathrm{~b}$. Furthermore, by using this system, a quick method of specular reflectivity becomes possible with the GISAXS measurement. Figure $4 \mathrm{~d}$ also shows the schematic view of a quick XR measurement with this soller system.

\section{RESULTS AND DISCUSSION}

Instrumental capabilities used with the initial test experiments GISAXS measurements. Figure 5 shows the GISAXS patterns obtained for the thin films of polystyrene-block-polymetyl methacrylate (PS- $b$-PMMA) at the incident angle of $0.15^{\circ}$. The $q$ range covers from 0.045 to $1.40 \mathrm{~nm}^{-1}$ in the GISAXS measurements at $\lambda=1.0 \AA$. The full-width at half-maximum of the beam at the detector position is $100 \times 30 \mu \mathrm{m}^{2}$ (horizontal $\times$ vertical). The primary beam stop is a rectangular aluminum block with a lead plate with dimensions of $2.5 \mathrm{~mm}$ in the horizontal and $30 \mathrm{~mm}$ in the vertical direction. Here, the number-average molecular weight of PS- $b$-PMMA and the weight fraction of the PS in PS- $b$-PMMA are $47.7 \times 10^{3} \mathrm{~g} \mathrm{~mol}^{-1}$ and $74.4 \%$, respectively. A thin film with approximately $110 \mathrm{~nm}$ thickness was spin-cast from a $2 \mathrm{wt} \%$ solution of PS- $b$-PMMA in the solvent toluene onto an Si-wafer at 2000 r.p.m. Then, we annealed the as-spun film for $1 \mathrm{~h}$ at $200^{\circ} \mathrm{C}$. We measured the changes in the two-dimensional GISAXS patterns, associated with the structural ordering of PS- $b$-PMMA, during the thermal annealing process using a CCD with an image intensifier detector. In the GISAXS pattern for the as-spun film (Figure 5a), a Bragg peak indicating the structural ordering of PS- $b$-PMMA in the as-spun film was not observed clearly. On the other hand, streaks along the $q_{\mathrm{Z}}$ direction were observed for the annealed sample (Figure 5b). Moreover, a higher order peak also appeared in the annealed sample (indicated by arrows in Figure 5b). These results show that a cylindrical structure aligned perpendicular to the film surface grows during the thermal annealing process and that the cylindrical structures form a hexagonal lattice with a $\mathrm{d}$-spacing of $27.3 \mathrm{~nm}$. This result agrees with the atomic force microscopy pattern of the thin film as shown in Figure $5 c$. It is well known that the block copolymers form a microdomain structure that have a $10-100 \mathrm{~nm}$ periodicity, with long-range order. In the as-spun state of the PS- $b$-PMMA, quick evaporation prohibits the ordering due to vitrification. During the thermal annealing process above the glass transition temperature, the PS- $b$-PMMA can self-assemble into the cylindrical microdomain structure. In a thin film, the specific surface area is much larger than in the bulk state, so the interfacial energy at the free surface and substrate strongly affects the orientation of the microdomain structure. Usually, the affinity of both components of the block copolymer to air or the substrate ( $\mathrm{Si}$ ) is different, and the difference induces a cylindrical structure parallel to the film surface. The parallel cylinder can reduce the interfacial free energy, although the conformational entropy may become unfavorable. In the case of the PS- $b$-PMMA, the surface free energy of PS to air is almost identical with that of PMMA; therefore, the cylindrical structure is perpendicular to the film surface because the conformational entropy becomes dominant for this orientation.

GIWAXS measurements. Figures $6 \mathrm{a}$ and $\mathrm{b}$ show the GIWAXS images of the poly(2-(perfluorooctyl)ethyl acrylate) (designated as PFA-C $\mathrm{C}_{8}$ ) thin film measured by the IP detector for only $5 \mathrm{~s}$. The full-width at half-maximum of the direct beam at the sample position was approximately $200 \times 30 \mu \mathrm{m}^{2}$ (horizontal $\times$ vertical) at $\lambda=1.0 \AA$, and the camera length was $476 \mathrm{~mm}$, calibrated with silver behenate at the incident angles of $0.08^{\circ}$ and $0.16^{\circ}$. A thin film with a thickness of approximately $100 \mathrm{~nm}$ was spin-cast from a $1 \mathrm{wt} \%$ solution of $\mathrm{PFA}-\mathrm{C}_{8}$ in the solvent AK-225 (Asahi Glass, Tokyo, Japan) onto a 1 in Si-wafer at 2000 r.p.m. for $30 \mathrm{~s}$.

The PFA- $\mathrm{C}_{8}$ formed a smectic $\mathrm{B}$ phase in which relatively rigid perfluorooctyl side chains were segregated from the main chain and the alkyl side chain and worked as mesogens. In both Figures $6 \mathrm{a}$ and

Table 1 Specifications of each measurement at $\lambda=1.0 \AA$

\begin{tabular}{|c|c|c|c|c|c|c|c|}
\hline & Detector & Camera length $(\mathrm{m})$ & Effective pixel & $\mathrm{q}$-range $\left(n m^{-1}\right)$ & $\Delta \mathrm{q} / \mathrm{q}$ & $\theta$-range $\left(^{\circ}\right)$ & $\Delta \theta\left(^{\circ}\right)$ \\
\hline \multirow[t]{7}{*}{ GISWAXS } & \multirow[t]{3}{*}{ CCD } & 0.5 & \multirow[t]{3}{*}{$1344 \times 1024$} & $0.19 \sim 6.0$ & $4.2 \times 10^{-2}$ & & \\
\hline & & 1.0 & & $0.09 \sim 3.0$ & $4.2 \times 10^{-2}$ & & \\
\hline & & 2.0 & & $0.05 \sim 1.5$ & $4.0 \times 10^{-2}$ & & \\
\hline & \multirow[t]{3}{*}{ IP } & 0.5 & \multirow[t]{3}{*}{$3000 \times 3000$} & $0.19 \sim 25.0$ & $6.7 \times 10^{-2}$ & & \\
\hline & & 1.0 & & $0.09 \sim 12.5$ & $6.7 \times 10^{-2}$ & & \\
\hline & & 2.0 & & $0.05 \sim 6.2$ & $6.0 \times 10^{-2}$ & & \\
\hline & FPD & 0.07 & $1032 \times 1032$ & $5 \sim 30$ & $9.0 \times 10^{-3}$ & & \\
\hline
\end{tabular}

\begin{tabular}{llr} 
GIXD & Scintillator & $1 \sim 72$ \\
XR & Scintillator +PIN-photodiode & 0.099 \\
Quick XR & PIN-photodiode & 0.0096 \\
\hline
\end{tabular}

Abbreviations: CCD, charge-coupled device; FPD, flat panel detector; GISWAXS, grazing incidence small/wide-angle X-ray scattering; GIXD, grazing incidence X-ray diffraction; IP, imaging plate; PIN, positive-intrinsic-negative; XR, X-ray reflectivity. 
a
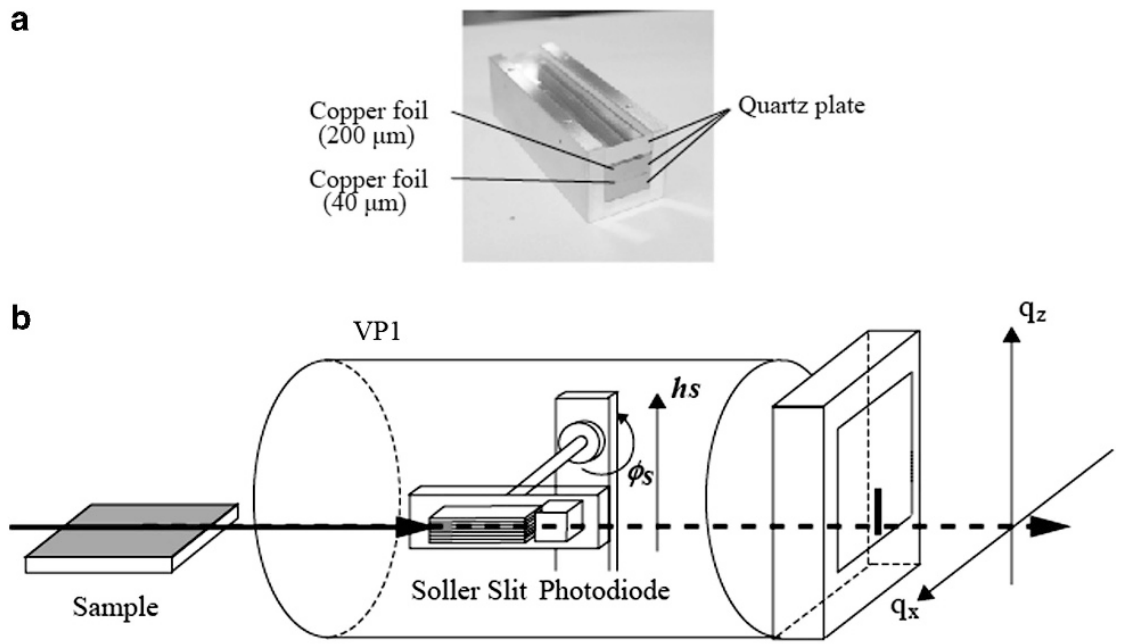

C

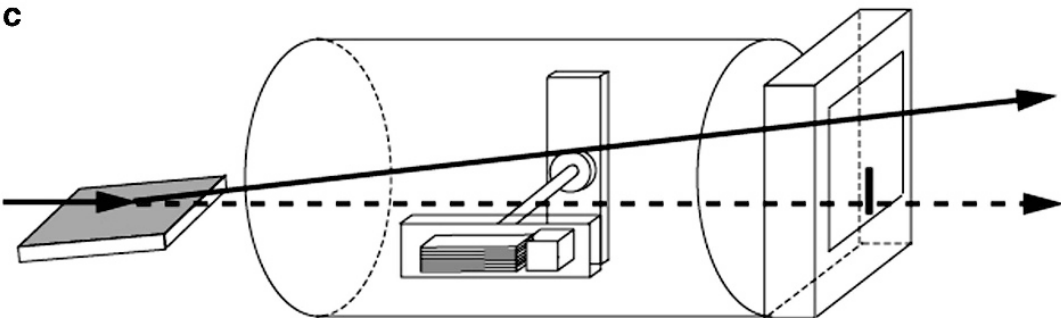

d

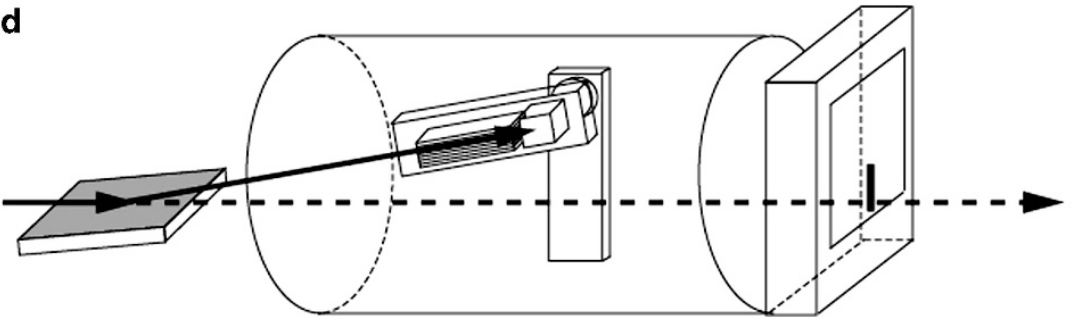

Figure 4 (a) Photograph and sketch for the position of the soller-slit and positive-intrinsic-negative positive-intrinsic-negative (PIN)-photodiode system in the VP1 for (b) the grazing incidence small/wide-angle X-ray scattering (GISAXS) measurement, (c) the sample alignment and (d) the quick X-ray reflectivity (XR) measurement.
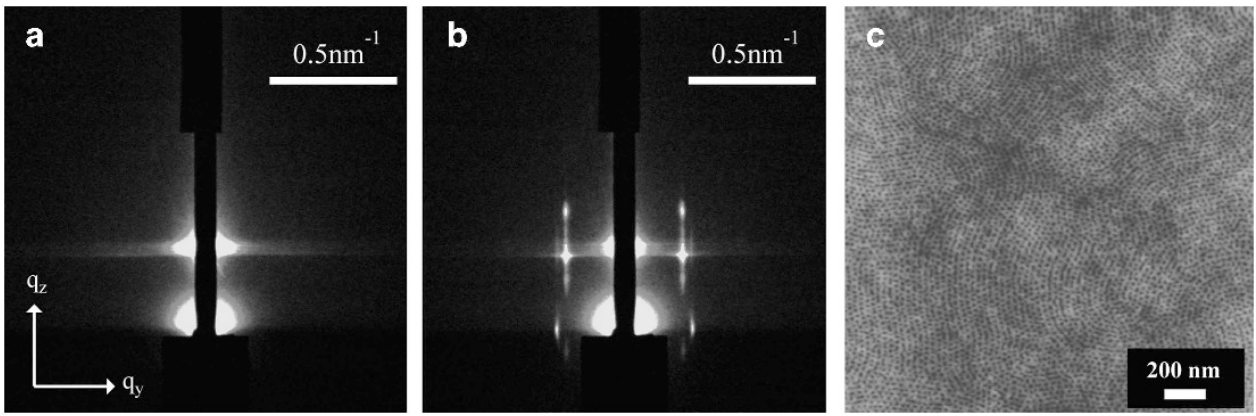

Figure 5 Time change in the grazing incidence small/wide-angle X-ray scattering (GISAXS) patterns of a polystyrene-block-polymetyl methacrylate (PS- $b$ PMMA) thin film (a) as-spun and (b) after $1 \mathrm{~h}$ annealing at $200^{\circ} \mathrm{C}$. A atomic force microscopy image of the PS- $b$-PMMA thin film after $1 \mathrm{~h}$ annealing at $200^{\circ} \mathrm{C}$ is shown in (c). A full color version of this figure is available at Polymer Journal online. 

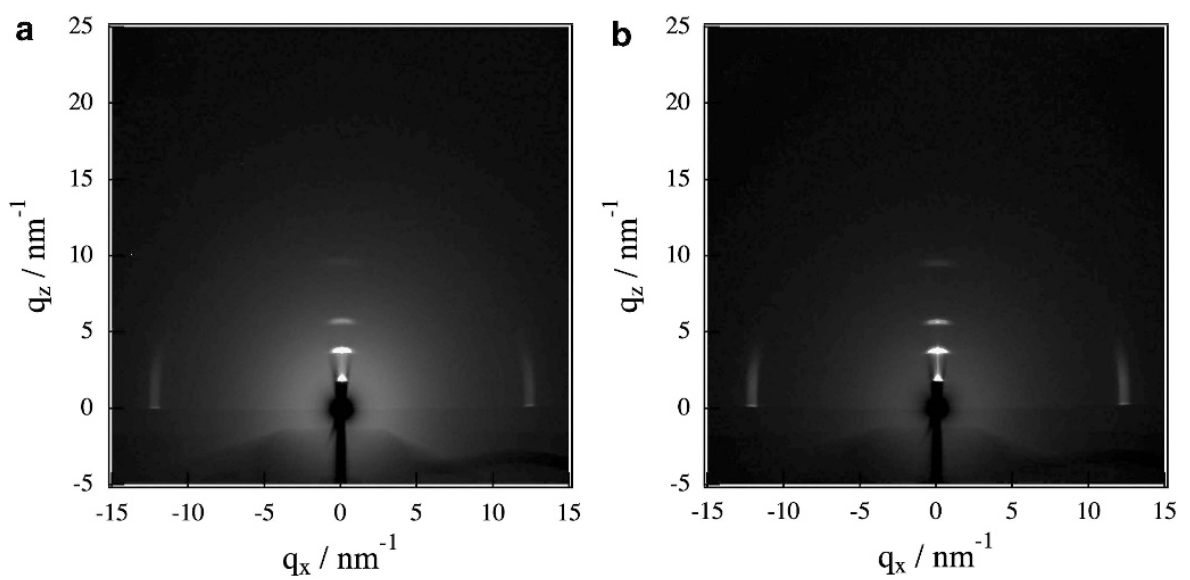

Figure 6 Grazing incidence wide-angle x-ray scattering (GIWAXS) images obtained for the poly(2-(perfluorooctyl)ethyl acrylate) (PFA- $C_{8}$ ) thin film with an incident angle of $0.08^{\circ}$ (a) and $0.16^{\circ}$ (b) at $\lambda=1.0 \AA$.
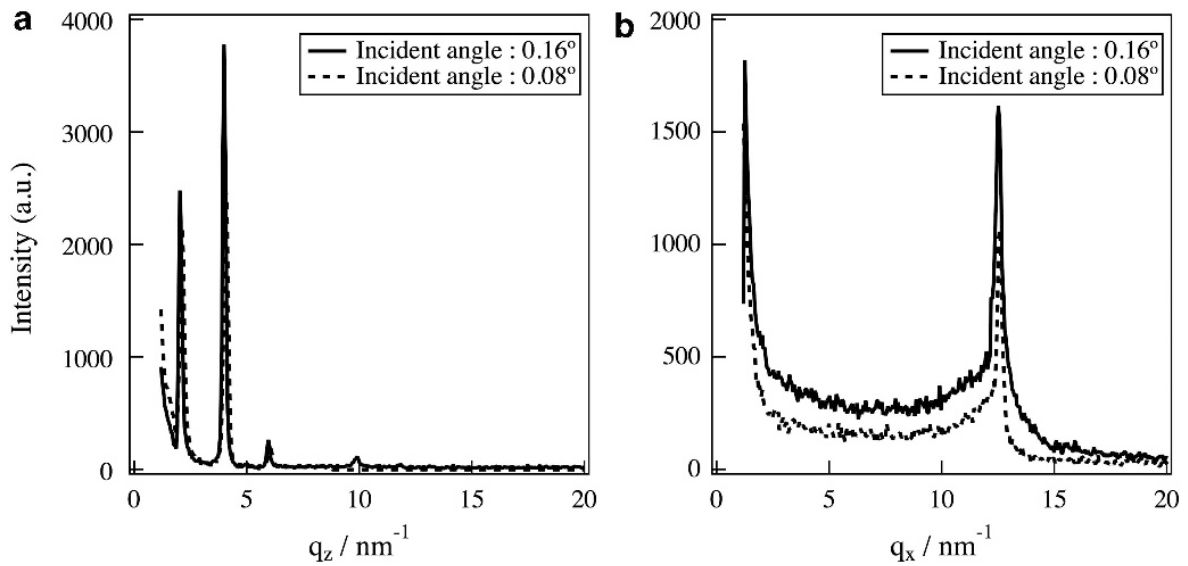

Figure 7 Grazing incidence X-ray diffraction (GIXD) intensity profiles in (a) the out-of-plane and the in-plane direction (b) for the poly(2-(perfluorooctyl)ethyl acrylate) (PFA- $C_{8}$ ) thin film at $\lambda=1.0 \AA$. The solid lines represent profiles for the incident angle of $0.16^{\circ}$ and the dashed lines represent profiles for $0.08^{\circ}$.

b, four sharp diffraction peaks appeared along the $q_{\mathrm{Z}}$ direction (out-of-plane), and a somewhat broad diffraction peak appeared along the $q_{\mathrm{X}}$ direction (in-plane). The out-of-plane diffraction peaks correspond to the first-, second-, third- and fifth-order diffraction from the layer of the smectic B phase, and the in-plane diffraction peak corresponds to the lateral spacing between the perfluorooctyl side chains in the smectic layer. The spacing of the first-order layer diffraction peak was estimated as $3.15 \mathrm{~nm}$ $\left(q_{\mathrm{Z}}=2.01 \mathrm{~nm}^{-1}\right)$, and the spacing of the lateral diffraction peak was $0.50 \mathrm{~nm}\left(q_{\mathrm{X}}=12.5 \mathrm{~nm}^{-1}\right)$.

GIXD measurements. Figures $7 \mathrm{a}$ and $\mathrm{b}$ show the GIXD intensity profiles along the out-of-plane and the in-plane directions, respectively, for the $\mathrm{PFA}-\mathrm{C}_{8}$ thin film. The sample and experimental condition of the incident angle $\left(0.08^{\circ}\right.$ and $\left.0.16^{\circ}\right)$ were the same as those for the GIWAX measurements (as in GIWAXS measurements section). The sharp layer diffraction peaks along the out-of-plane direction and the broad diffraction peak along the in-plane direction can be measured with a higher $\mathrm{S} / \mathrm{N}$ ratio compared with those in the GIWAS measurements. These results are difficult to detect using a labscale WAXD instrument.
Quick XR measurement. Using the quick XR system, we measured a reflection profile of the polystyrene thin film on an Si-wafer, which is a typical amorphous polymer thin film (Figure 8 ). The incident X-ray wavelength was $1.0 \AA$, and the $q$ range covered in the present measurements was from 0.06 to $0.3 \AA^{-1}$, with a divergence angle of $0.164^{\circ}$. The total acquisition time for the measurement is approximately $10 \mathrm{~min}$ with $800 \mathrm{~ms}$ per point. The XR profile was fit based on the well-known Parratt algorithm ${ }^{14}$ for a single layer model on an Si-wafer with a native oxide layer. In this model, the surface and the interface roughness were defined by an error function, as modified by Névot and Croce. ${ }^{15}$ The estimated thickness and surface roughness were 362 and $3 \AA$, respectively.

$X R$ measurements. The precise XR measurements were performed using a vertical geometry with a $\theta_{\mathrm{H}}^{\prime}=2 \theta_{\mathrm{H}}$ relation. The reflected beam was collimated by the two-detector-slit system on the detector arm. Figure 9 shows the reflectivity profile of the amorphous polystyrene film at room temperature, which is for the same sample as the one used for the quick XR measurement (as in GIXD measurements section). The reflectivity profile was measured by the PIN-photodiode for $\theta_{\mathrm{H}}^{\prime}<0.5^{\circ}$ and by the scintillator for $\theta_{\mathrm{H}}^{\prime}>0.5^{\circ}$. The total acquisition time for the measurement was approximately 


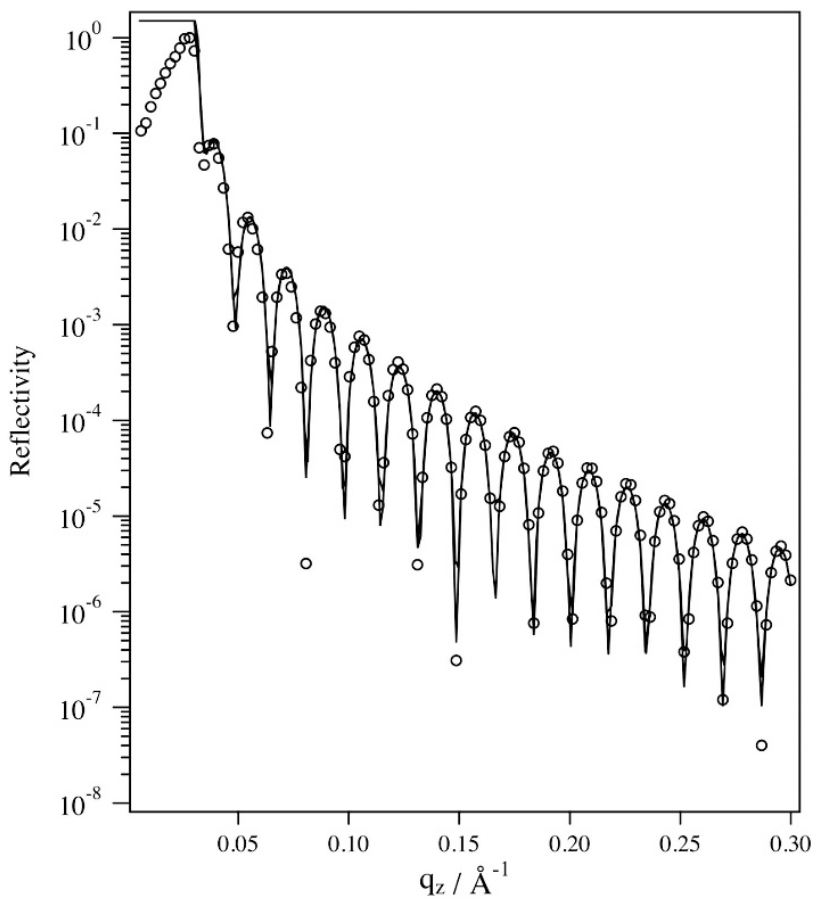

Figure $8 \mathrm{X}$-ray reflectivity (XR) profile of the polystyrene thin film on a silicon ( $\mathrm{Si}$ ) wafer (dots) using the quick XR measurement at $\lambda=1.0 \AA$. The solid curves are the results of a fit based on the Parratt algorithm.

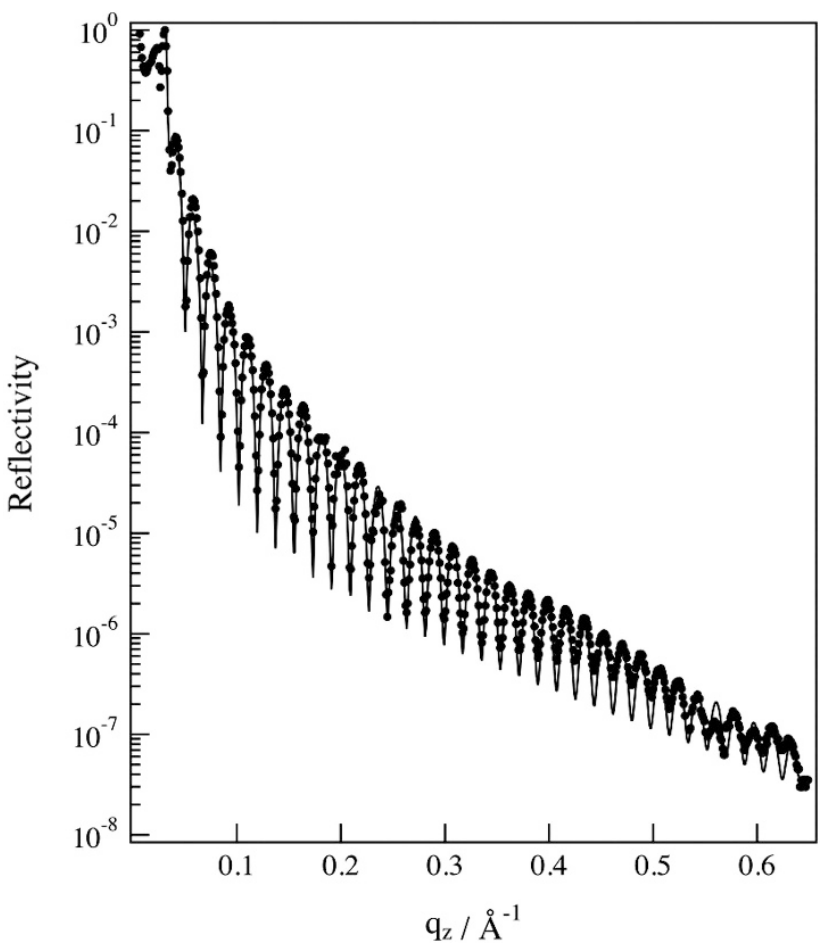

Figure 9 X-ray reflectivity (XR) profile of the polystyrene thin film on a silicon (Si) wafer (dots) using the $\theta_{\mathrm{H}}$ and $\theta_{H}^{\prime}$ scans at $\lambda=1.0 \AA$. The solid curves are the results of a fit based on the Parratt algorithm.
10 min with $800 \mathrm{~ms}$ per point. The XR profile was analyzed by fitting a single layer model on an Si-wafer with a native oxide layer. A calculated reflectivity based on the single layer model (solid line in Figure 9) gave good agreement with the observed one (open circle in Figure 9). As a result, the structural parameters were estimated as a layer thickness of $348 \AA$ and a surface roughness of $3 \AA$, which are almost the same as the results from the quick XR measurement. The advantage of this measurement is that a dynamic range up to eight orders of magnitude can be measured.

\section{CONCLUSIONS}

We have constructed a 'nanoscopic surface and interface measurement system' in the first hutch of the BL03XU FSBL at SPring-8. Using this integrated system, we can measure the hierarchical structure of the same sample. The first hutch is now under operation for GISWAXS-, GIXD- and XR-compatible measurements with a high photon flux of approximately $10^{13}$ photons per $\mathrm{s}$ and a low divergence. In the GISWAXS setup, it is possible to take simultaneous or individual measurements using II + CCD (or IP) detectors and a flat panel detector with an accessible $q$-range from 30 to $0.05 \mathrm{~nm}^{-1}$. In addition to the GISWAXS measurement, the quick XR measurement system can be used with a high dynamic range, with measurements up to six orders of magnitude. For the GIXD measurements, structural characterization of the in-plane and the out-of-plane directions can cover from $0^{\circ}$ to $70^{\circ}$ without changing the sample setting. Precise XR measurements can be performed with a high dynamic range of measurements, with up to eight orders of magnitude. For all modes of GISWAXS, GIXD and XR, measurements on a typical sample demonstrated the high capability and potential of the versatile diffractometer to characterize soft-material thin film samples.

\section{CONFLICT OF INTEREST}

The authors declare no conflict of interest.

\section{ACKNOWLEDGEMENTS}

We would like to acknowledge the 19 corporations comprising the FSBL consortium that funded the FSBL construction: Asahi Kasei; Kwansei Gakuin University; Canon; Kuraray; Showa Denko KK; Sumitomo Chemical; Sumitomo Rubber Industries; Sumitomo Bakelite; Denso; Toyobo; Toray Industries; Nitto Denko; Bridgestone; Mitsui Chemicals; Mitsubishi Chemical; Mitsubishi Rayon; Yokohama Rubber; Teijin; and DIC. We also thank to the many academic members who participated in the construction of the FSBL, and we acknowledge Dr R Ishige for providing the GIWAXS and GIXD measurement results. We also acknowledge Dr O Sakata (Nation Institute for Material Science) and Dr S Kimura (JASRI) for fruitful discussions in the conceptual design stage.

1 Tanaka, K., Yoon, J., Takahara, A. \& Kajiyama, T. Ultrathinning-induced surface phase separation of polystyrene/poly(vinyl mether ether) blend film. Macromolecules 28, 934-938 (1995).

2 Yamaguchi, H., Kikuchi, M., Kobayashi, M., Ogawa, H., Masunaga, H., Sakata, O. \& Takahara, A. Influence of molecular weight dispersity of poly\{2-(perfluorooctyl) ethyl acrylate\} brushes on their molecular aggregation states and wetting behavior. Macromolecules 45, 1509-1516 (2012).

3 Morita, H., Yamada, M., Yamaguchi, T. \& Doi, M. Molecular dynamics study of the adhesion between end-grafted polymer films. Polym. J. 37, 782-788 (2005).

4 Reiter, G. Dewetting as a probe of polymer mobility in thin films. Macromolecules 27, 3046-3052 (1994).

5 Ogawa, H., Kanaya, T., Nishida, K. \& Matsuba, G. Phase separation and dewetting in polystyrene/poly(vinyl mether ether) blend thin films in a wide thickness range. Polymer 49, 254-262 (2008).

6 Kanaya, T., Inoue, R., Kawashima, K., Miyazaki, T., Tsukushi, I., Shibata, K., Matsuba, G., Nishida, K. \& Hino, M. Glassy dynamics and heterogeneity of polymer thin films. J. Phys. Soc. Jpn 78, 041004-1-041004-9 (2009).

7 Miyazaki, T., Nishida, K. \& Kanaya, T. Contraction and reexpansion of polymer thin films. Phys. Rev. E 69, 022801-1-022801-4 (2004). 
8 Yokoyama, H., Dutriez, C., Li, L., Nemoto, T., Sugiyama, K., Sasaki, S., Masunaga, M., Takata, M. \& Okuda, H. Grazing incident small angle x-ray scattering study of polymer thin films with embedded ordered nanometer cells. J. Chem. Phys. 127, 014904-1014904-9 (2007)

9 Okuda, H., Kato, M., Kuno, K., Ochiai, S., Usami, N., Nakajima, K. \& Sakata., O. A grazing incidence small-angle x-ray scattering analysis on capped Ge nanodots in layer structures. J. Phys. 22, 474003-1-474003-7 (2010).

10 Lee, B., Park, I., Jinhwan, Y., Park, S., Kim, J., Kim, K., Chang, T. \& Ree, M. Structural analysis of block copolymer thin films with grazing incidence small angle x-ray scattering. Macromolecules 38, 4311-4323 (2005).

11 Masunaga, H., Ogawa, H., Takano, T., Sasaki, S., Goto, S., Tanaka, T., Seike, T., Takahashi, S., Takashita, K., Nariyama, N., Ohashi, H., Ohta, T., Furukawa, Y., Matsushita, T., Ishizawa, Y., Yagi, N., Takata, M., Kitamura, H., Sakurai, K., Tashiro, K., Takahara, A., Amemiya, Y., Horie, K., Takenaka, M.,
Kanaya, T., Jinnai, H., Okuda, H., Akiba, I., Takahashi, I., Yamamoto, K., Hlkosaka, M., Sakurai, S., Shinohara, Y., Okada, A. \& Sugihara, Y. Multipurpose soft-material SAXS/WAXS/GISAXS beamline at SPring-8. Polym. J. 43, 431-477 (2011).

12 Amemiya, Y., Ito, K., Yagi, N., Asano, Y., Wakabayashi, K., Ueki, T. \& Endo, T. Largeaperture TV detector with a beryllium-windowed image intensifier for x-ray diffraction. Rev. Sci. Instrum. 66, 2290-2294 (1995).

13 Yagi, N. \& Inoue, K. CMOS flatpanel detectors for SAXS/WAXS experiments. J. Appl. Crystallogr. 40, s439-s441 (2007).

14 Parratt, L.G. Surface studies of solids by total reflection of x-rays. Phys. Rev. 95, 359-369 (1954)

15 Nevot, L. \& Croce, P. Caracterisation des surfaces par reflexion rasante de rayons $X$ Application a l'etude du polissage de quelques verres silicates. Rev. Phys. Appl. 15, 761-769 (1980). 